\title{
The canonical decomposition of once-punctured torus bundles
}

\author{
Marc Lackenby
}

\begin{abstract}
We determine the canonical polyhedral decomposition of every hyperbolic oncepunctured torus bundle over the circle. In fact, we show that the only ideal polyhedral decomposition that is straight in the hyperbolic structure and that is invariant under a certain involution is the ideal triangulation defined by Floyd and Hatcher. Unlike previous work on this problem, the techniques we use are not geometric. Instead, they involve angled polyhedral decompositions, thin position and a version of almost normal surface theory.
\end{abstract}

Mathematics Subject Classification (2000). 57N10, 57M50.

Keywords. Hyperbolic, 3-manifold, canonical polyhedral decomposition, once-punctured torus bundle.

\section{Introduction}

Epstein and Penner proved that any complete, non-compact, finite volume hyperbolic manifold admits a canonical decomposition into hyperbolic ideal polyhedra [2]. This is an important construction, particularly in dimension three, and yet it remains mysterious. Although it can be computed in practice for any particular hyperbolic 3-manifold, as demonstrated by the computer program SnapPea [11], it is difficult to determine the decomposition of an infinite family of examples. In this paper, we do exactly that for once-punctured torus bundles over the circle that admit a hyperbolic structure. They have a natural ideal triangulation, first constructed by Floyd and Hatcher [3], which we term the monodromy ideal triangulation. It is described in detail in $\S 2$ of the paper. Our main result is the following.

Theorem 1. The canonical polyhedral decomposition of a hyperbolic once-punctured torus bundle over the circle is its monodromy ideal triangulation.

Hyperbolic structures on once-punctured torus bundles were first studied by Jørgensen. He produced an unpublished manuscript on quasi-Fuchsian once- 


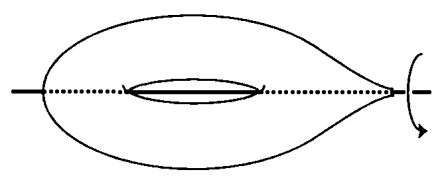

Figure 1.

punctured torus groups [5], but this did not deal with bundles. More recently, Akiyoshi, Sakuma, Wada and Yamashita have developed his methods further [1], and Akiyoshi has a programme for dealing with the bundle case. All these approaches have been highly geometric. Our techniques, however, are much more topological. They draw on Gabai's concept of thin position [4] and almost normal surface theory, due to Rubinstein [8], Thompson [10] and Stocking [9].

A once-punctured torus admits an involution, as shown in Figure 1, which commutes with any linear homeomorphism of the once-punctured torus. Hence, it induces a fibre-preserving involution of any once-punctured torus bundle over the circle. The canonical decomposition is preserved by this involution. We will prove the following stronger version of Theorem 1.

Theorem 2. Any ideal polyhedral decomposition of a hyperbolic once-punctured torus bundle that is straight in the hyperbolic structure and that is invariant under the fibre-preserving involution is equivariantly isotopic to the monodromy ideal triangulation.

The assumption that the decomposition is invariant under this involution is vital. For example, the figure-eight knot complement, which is a once-punctured torus bundle, admits several straight ideal triangulations, as follows. Start with the canonical decomposition into two ideal tetrahedra. Remove a common face, forming a single ideal polyhedron. This can then be decomposed into three ideal tetrahedra arranged around an edge, which form another straight ideal triangulation of the knot complement.

A key concept in this paper is that of an angled polyhedral decomposition of a 3-manifold $M$. A polyhedron is a 3-ball with a specified graph in its boundary, which must satisfy the following conditions:

- each vertex has valence at least three;

- no edge is a loop;

- each complementary region is a disc with at least three edges in its boundary. An ideal polyhedron is a polyhedron with its vertices removed. The resulting punctures are termed ideal vertices. An ideal polyhedral decomposition of a 3-manifold $M$ is a decomposition of $M-\partial M$ into ideal polyhedra, with faces glued homeomorphically in pairs. An angled polyhedral decomposition is an ideal polyhedral decomposition, together with an assignment to each edge of each ideal polyhedron of an interior and exterior angle in the range $(0, \pi)$, which sum to $\pi$. These must 
satisfy the following conditions:

- the sum of the interior angles around each edge is $2 \pi$;

- for any closed curve in the boundary of an ideal polyhedron that misses the ideal vertices, that intersects each edge transversely at most once and that does not lie wholly in a face, the sum of the exterior angles of the edges it runs over is at least $2 \pi$, with equality if and only it it encircles an ideal vertex.

The second of the above conditions is, by Rivin's theorem, exactly the requirement that an ideal polyhedron with a given convex angle assignment to its edges be realizable in hyperbolic space [7]. However, the hyperbolic structures on these ideal polyhedra need not match up correctly under the face identifications, and therefore an angled polyhedral decomposition is significantly more general than a decomposition of a hyperbolic 3-manifold into straight ideal polyhedra. A similar concept (an angled spine) was introduced in [6]. We will in fact prove the following stronger version of Theorem 2 .

Theorem 3. Any angled polyhedral decomposition of a once-punctured torus bundle that is invariant under the fibre-preserving involution is equivariantly ambient isotopic to the monodromy ideal triangulation.

The strategy of the proof is to place the 1-skeleton of $M$ in 'thin position' in the fibration. We establish that either all the edges can be simultaneously equivariantly isotoped into fibres, or one of the fibres can be placed in a position that resembles almost normal form. We term this fairly normal form, and it seems to arise naturally when a 3-manifold admits certain sweep-outs by surfaces with non-empty boundary. As in [6], it is possible to assign an 'area' to this fairly normal surface $F$, which is defined additively over the discs of intersection with the ideal polyhedra, and which turns out to be $-2 \pi \chi(F)$. However, using the fact that $F$ is invariant under the involution and the fact that $F$ lies in a thick region of thin position, we deduce that some disc area has more than $2 \pi$ or two discs have area more than $\pi$. It is simple to prove that each disc has non-negative area. This gives a contradiction, since the Euler characteristic of a once-punctured torus is -1 . We therefore deduce that all the edges of the ideal polyhedral decomposition can be made simultaneously level in the fibration. With some further work, this implies that it is the monodromy ideal triangulation.

I would like to thank Brian Bowditch for introducing me to this problem. I would also like to thank Makoto Sakuma who gave a talk on his work with Akiyoshi, Wada and Yamashita [1] on quasi-fuchsian once-punctured torus groups, and the canonical decomposition of two-bridge link complements. It seems likely that the techniques of this paper can be applied to two-bridge links. 


\section{The monodromy ideal triangulation}

This section contains a description of the monodromy ideal triangulation defined by Floyd and Hatcher [3]. The once-punctured torus $F$ can be constructed from two ideal triangles by gluing their sides in pairs, and any ideal triangulation of $F$ takes this form. Floyd and Hatcher found a very elegant way of encoding the set of isotopy classes of such ideal triangulations as the vertices of a tree.

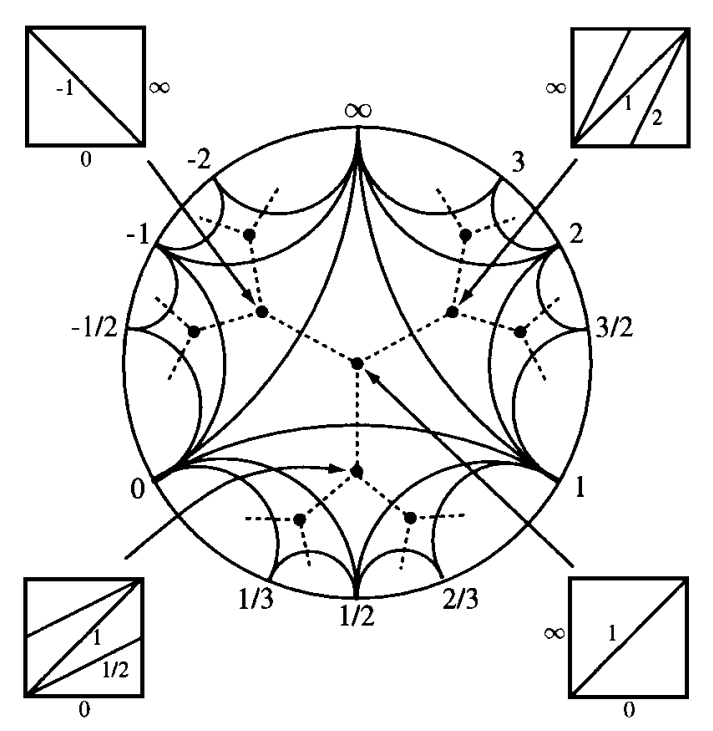

Figure 2.

This tree is dual to a tessellation of the hyperbolic plane by ideal triangles. The ideal vertices of this tessellation are $\mathbb{Q} \cup\{\infty\}$ in the circle at infinity. Associated to each ideal vertex, there is a properly embedded arc in $F$ with that slope. Two ideal vertices are joined by a geodesic if and only if the corresponding arcs can be isotoped off each other. These geodesics divide the hyperbolic plane into ideal triangles, forming the required tessellation, which is known as the diagram of $P S L(2, \mathbb{Z})$. (See Figure 2.) The ideal vertices of an ideal triangle correspond to three disjoint non-parallel properly embedded arcs in $F$, and hence an ideal triangulation. Thus, there is one vertex of the dual tree for each isotopy class of ideal triangulation of the once-punctured torus. Two vertices of the tree are joined by an edge if and only if their corresponding ideal triangulations differ by an elementary move, which involves removing one of the edges, resulting in a square with side identifications, and then inserting the other diagonal of the square.

The monodromy of a once-punctured torus bundle induces a homeomorphism 


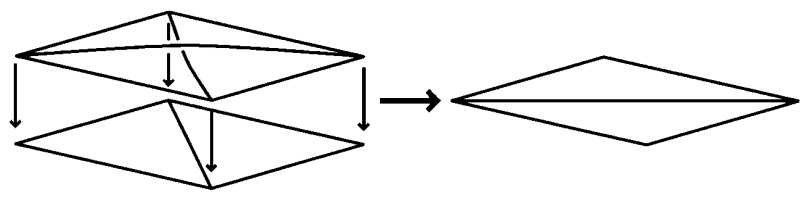

Figure 3.

of this tree. Any homeomorphism of a simplicial tree either has a fixed point or leaves invariant a unique copy of $\mathbb{R}$, known as the axis. In the former case, the monodromy is periodic, and hence the bundle is not hyperbolic. In the latter case, we pick a vertex on the axis. The unique path in the tree from this vertex to its image under the monodromy homeomorphism runs along the axis. It specifies a sequence of elementary moves. These induce an ideal triangulation of the bundle, as follows. Start with the once-punctured torus with its initial ideal triangulation. Realize the first elementary move by attaching an ideal triangulation to the oncepunctured torus, as shown in Figure 3. Continue in this fashion along the path in the graph as far as the final vertex. We then glue the top and bottom ideal triangulations via the monodromy. The result is the monodromy ideal triangulation of the bundle.

\section{Fairly normal surfaces}

It is a famous result, due to Rubinstein [8] and Stocking [9], that a strongly irreducible Heegaard surface of a compact orientable 3-manifold can be placed in almost normal form in any given triangulation. The arguments rely heavily on Gabai's concept of thin position [4]. Many of these techniques generalise to other settings: bridge decompositions of link complements, and surface fibrations over the circle. However, it does not seem to be possible in general to deduce that the relevant surface (be it a bridge punctured 2-sphere or a fibre) can be placed in almost normal form. But there is a weaker version of the theory which we now introduce.

Suppose we are given an ideal polyhedral decomposition of a compact orientable 3-manifold with non-empty boundary. Truncate the ideal vertices, making the polyhedra compact. These polyhedra now have two types of faces: interior faces, which are truncated copies of the original faces, and boundary faces which are links of ideal vertices. The edges of the truncated polyhedra also come in two types, boundary edges which lie in $\partial M$, and interior edges which we denote by $\Delta^{1}$. Note that no two interior edges are adjacent.

A properly embedded surface $F$ is fairly normal if it intersects each polyhedron in fairly normal discs. A fairly normal disc is a properly embedded disc with the following properties: 

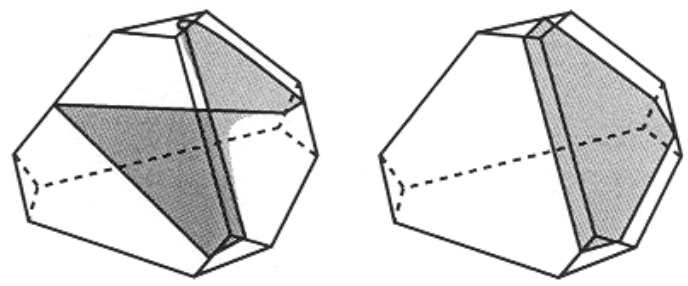

Figure 4

- it intersects any boundary face in at most one arc;

- it intersects any interior face in arcs, that each starts and ends in distinct non-adjacent edges of the face;

- it intersects any interior edge at most twice;

- it is not parallel to a disc in the boundary of the polyhedron disjoint from the interior edges.

A weakly normal disc is a properly embedded disc satisfying the first two of the above conditions. Note that in any given polyhedron, there are only finitely many fairly normal disc types, but they are far more numerous than normal discs. Some examples of fairly normal discs in truncated tetrahedra are shown in Figure 4.

We will show that, when $M$ fibres over the circle, and when the ideal polyhedral decomposition is angled, then either there is an ambient isotopy taking each interior edge into a fibre, or some fibre can be placed in fairly normal form. This is nothing new: fairly normal surfaces are a generalisation of normal surfaces, and it is well known that a fibre can be ambient isotoped into normal form. However, the fairly normal surface will have a number of extra properties, which will eventually lead to a contradiction in the case of a once-punctured torus. For example, it will have the maximal number of intersection points with $\Delta^{1}$ over all fibres not containing level edges.

When the ideal polyhedral decomposition is angled, it is possible to assign an area to weakly normal (and more general) discs. This is the sum of the exterior angles of the interior edges the disc runs over (counted with multiplicity), plus the number of arcs of intersection with the boundary faces multiplied by $\pi$, then with $2 \pi$ subtracted. The area of a fairly normal surface $F$ is the sum of the areas of its discs. It is shown in $[6]$ that the area of a properly embedded surface $F$ is equal to $-2 \pi \chi(F)$.

Many of the fairly normal surfaces $F$ we will consider will have a face compression disc which we define to be a disc $D$ embedded in a polyhedron $P$, such that

- $D$ is disjoint from $\Delta^{1}$;

- the interior of $D$ is disjoint from $\partial P \cup F$;

- the boundary of $D$ is an $\operatorname{arc}$ in $F$, an arc in an interior face and possibly an arc in a boundary face;

- the arc $\partial D \cap \partial P$ is not parallel in $\partial P-\Delta^{1}$ to a subarc of $F \cap \partial P$. 
For example, both the fairly normal discs shown in Figure 4 have face compression discs.

If a weakly normal disc is surgered along a face compression disc, the result is two discs that need not be weakly normal. For they may have an arc of intersection with an interior face that has endpoints

- in the same boundary edge,

- in the same interior edge, or

- in adjacent interior and boundary edges.

In the first case, the endpoints of this arc must close up in the boundary face to form a closed curve that is disjoint from the interior edges and that intersects the boundary faces in only one arc. We call such a disc boundary-trivial.

In the second and third cases, there is an obvious isotopy of the discs that reduces the number of intersections with the interior edges. We may repeat this procedure until we end with discs that are boundary-trivial or weakly normal. Note that in the third case, we always end with a weakly normal disc.

We now define a certain type of fairly normal disc in a polyhedron $P$. Let $\alpha$ be either an interior edge of $P$ or an arc properly embedded in an interior face with endpoints in distinct boundary edges. Let $\mathcal{N}(\alpha)$ be a small regular neighbourhood of $\alpha$ in $\partial P$, and let $D$ be a properly embedded disc with boundary $\partial \mathcal{N}(\alpha)$. Then $D$ is a weak bigon. It is a bigon if $\alpha$ was an interior edge. Note that a fairly normal weak bigon is a bigon.

Lemma 4. Let $D$ be a weakly normal disc. Then the area of $D$ is non-negative. The area is zero if and only if it is the link of an ideal vertex or a weak bigon.

Proof. Consider first the case where $D$ is disjoint from the boundary faces. Suppose that it intersects some interior edge more than once. It then has a face compression disc disjoint from the boundary faces. If we surger along this disc, the result is two new discs. The area of $D$ is the sum of the areas of these two discs, plus $2 \pi$. If these discs fail to be weakly normal, there is an isotopy that reduces their area and that takes them to weakly normal discs. Thus, we may assume that $D$ intersects each interior edge at most once. But, then by the definition of an angled polyhedral decomposition, its area is non-negative and in fact is strictly positive unless the disc is the link of an ideal vertex.

Suppose now that $D$ intersects the boundary faces in a single arc. The interior edges emanating from the boundary face containing this arc have exterior angles that sum to $2 \pi$. Thus, there is a way of isotoping the arc off the boundary face so that the new points of intersection with the interior edges have total exterior angle at most $\pi$. So, the resulting disc has area at most that of $D$. It may fail to be weakly normal, in which case there is an isotopy which removes two intersection points with the interior edges. Repeat until we end with a weakly normal disc $D^{\prime}$. By the above argument, $D^{\prime}$ has non-negative area and hence so does $D$. If the area of $D$ is zero, then $D^{\prime}$ must have been the link of an ideal vertex. Also, 
$D$ would have been obtained from $D^{\prime}$ by isotoping $D^{\prime}$, without introducing any intersection points with interior edges, so that a single sub-arc of $\partial D$ runs over a boundary face. The only way of doing this is to isotope $\partial D^{\prime}$ along an interior edge or across an interior face. But in both cases, the resulting $D$ has positive area.

If $D$ intersects the boundary faces in more than two arcs, then the area is positive. If it intersects the boundary faces exactly two times, the area is nonnegative, and is in fact strictly positive unless it is disjoint from the interior edges. But in this case, we claim that $D$ is a weak bigon. For, as above, there is an isotopy of $D$ removing one of its arcs of intersections with the boundary faces without increasing its area. After a further isotopy that does not increase area, we end with a weakly normal or boundary-trivial disc. In the former case, this disc has positive area since it has only a single arc of intersection with the boundary faces. Hence $D$ also has positive area. In the latter case, $D$ was originally a weak bigon.

A consequence of the above lemma is that a fairly normal surface cannot be a sphere or a properly embedded disc. Also, by observing that bigons cannot be glued to links of ideal vertices, we see that a fairly normal surface with zero Euler characteristic is composed either entirely of vertex links or entirely of bigons. Hence, it is a boundary-parallel torus or a compressible annulus. This implies that a compact orientable 3-manifold with an angled polyhedral decomposition is irreducible, atoroidal, an-annular and has non-empty boundary consisting of tori. Its interior therefore admits a complete finite volume hyperbolic structure.

We now define some further types of fairly normal disc. Let $\alpha$ be an arc in the boundary of a polyhedron $P$ such that

- the interior of $\alpha$ is disjoint from the boundary faces;

- the endpoints of $\alpha$ lie on boundary edges of distinct boundary faces;

- the intersection of $\alpha$ with each interior face is a collection of arcs, each of which runs between distinct non-adjacent edges of the face;

- $\alpha$ intersects each interior edge at most once.

Let $\mathcal{N}(\alpha)$ be a small regular neighbourhood of $\alpha$ in $\partial P$, and let $D$ be a properly embedded disc with boundary $\partial \mathcal{N}(\alpha)$. Then $D$ is an arclike fairly normal disc, and $\alpha$ is its associated arc. An arclike disc in a truncated ideal tetrahedron is shown in Figure 5.

We give some further definitions. Let $D$ be a properly embedded disc parallel to a boundary face in a polyhedron $P$. Pick one or two disjoint $\operatorname{arcs} \alpha$ in $\partial P$, starting in $\partial D$ and running across an interior face to a boundary face. Ensure that each component of $\alpha$ is not parallel to a sub-arc of an interior edge, and that if $\alpha$ consists of two arcs, they are not parallel. Modify by $D$ by isotoping along $\alpha$. The result is a modified vertex link.

Similarly, let $D$ be two non-parallel ideal vertex links. Let $\alpha$ be an arc in an interior face, running between the two discs of $D$. Ensure that $\alpha$ is not parallel to a sub-arc of an interior edge. Modify $D$ by isotoping the boundaries of the two 


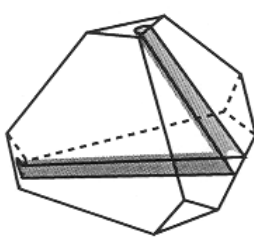

Arclike disc

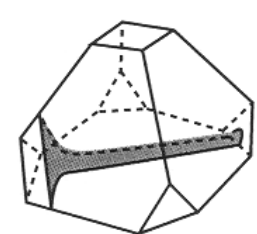

Modified vertex link

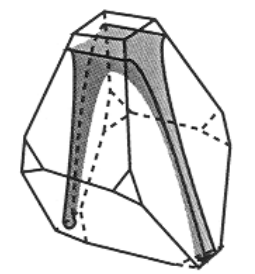

Modified vertex link

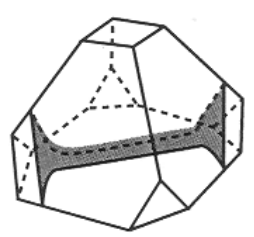

Fused vertex link

Figure 5.

discs towards each other along $\alpha$, and then fusing to form a single disc, which we term a fused vertex link.

Lemma 5. Let $D$ be a fairly normal disc in a polyhedron $P$. Suppose that $D$ has a face compression disc, and also that, if $D$ is arclike, the face compression disc does not lie on the same side as its associated arc. Then the area of $D$ is at least $\pi$. Moreover, if $D$ is invariant under an involution of $P$ that preserves the disc's orientation and transverse orientation, then it has area at least $2 \pi$. If either of these inequalities are equalities, then the disc must be disjoint from the interior edges or be a modified vertex link or a fused vertex link.

Proof. Surger $D$ along the face compression disc to give two discs $D_{1}$ and $D_{2}$ properly embedded in $P$. They need not be weakly normal, but after an isotopy that does not increase area, we end up with discs $D_{1}^{\prime}$ and $D_{2}^{\prime}$ that are weakly normal or boundary-trivial.

If $D_{1}^{\prime}$ and $D_{2}^{\prime}$ are both boundary-trivial, then $D$ was arclike and the face compression disc lay on the arc side, contrary to hypothesis. So, at least one $D_{i}^{\prime}$ is weakly normal, and so has non-negative area, by Lemma 4 . If either of $D_{1}^{\prime}$ or $D_{2}^{\prime}$ are boundary-trivial, then the face compression disc was disjoint from the boundary faces of $P$. In this case, the area of $D$ is equal to the area of $D_{1}$ plus the area of $D_{2}$ plus $2 \pi$, and hence is at least $\pi$. If both $D_{1}^{\prime}$ and $D_{2}^{\prime}$ are weakly normal, then they have non-negative area. The area of $D$ is obtained by adding the area of $D_{1}$ and $D_{2}$, and then adding either $\pi$ or $2 \pi$, depending on whether the face compression disc ran over a boundary face or not. Thus, we deduce that the area of $D$ is at least $\pi$.

Now suppose that this inequality is an equality. Then $D_{1}$ and $D_{2}$ must be equal to $D_{1}^{\prime}$ and $D_{2}^{\prime}$. If $D_{1}$, say, is boundary-trivial, then $D_{2}$ must be a weakly normal disc with zero area. By Lemma 4, it is either a vertex link or a weak bigon. Hence, $D$ is either a modified vertex link or disjoint from $\Delta^{1}$. If $D_{1}$ and $D_{2}$ are both weakly normal, then they must both have zero area, and the face compression disc must have run over a boundary face. So, $D_{1}$ and $D_{2}$ must be weak bigons and hence, in this case, $D$ is disjoint from $\Delta^{1}$.

We must now show that if $D$ is invariant under an involution that preserves its 
orientation and transverse orientation, then it has area at least $2 \pi$. The involution of $P$ must be a rotation of order two, which preserves each component of $P-D$. The involution takes the face compression disc to another. It is a straightforward matter to ensure that these two discs are disjoint after an equivariant ambient isotopy. If we now surger $D$ along both these discs, the result is three discs $D_{1}$, $D_{2}$ and $D_{3}$, where $D_{2}$ is attached to $D_{1}$ and $D_{3}$. So, $D_{1}$ and $D_{3}$ are swapped by the involution and $D_{2}$ is invariant. There is an equivariant isotopy that takes $D_{1}$ and $D_{3}$ to discs $D_{1}^{\prime}$ and $D_{3}^{\prime}$ that are weakly normal or boundary-trivial, and that takes $D_{2}$ to a disc $D_{2}^{\prime}$ that is weakly normal or that has boundary in a single interior face. We call the latter type of disc face-trivial. Note that not all the discs can be trivial, for then $D$ would be arclike and its face compression disc would have been on the arc side.

Consider first the case where the face compression discs are disjoint from the boundary faces of $P$. Then the area of $D$ is the sum of the areas of $D_{1}, D_{2}$ and $D_{3}$, plus $4 \pi$. If $D_{2}^{\prime}$ is face-trivial, its area is $-2 \pi$ and the areas of $D_{1}^{\prime}$ and $D_{3}^{\prime}$ are non-negative. If $D_{1}^{\prime}$ and $D_{3}^{\prime}$ are boundary-trivial, then their areas are $-\pi$, and the area of $D_{2}^{\prime}$ is non-negative. Thus, we deduce that the area of $D$ is at least $2 \pi$. The case where the face compression discs intersect the boundary faces of $P$ is easier. Each $D_{i}^{\prime}$ is weakly normal, and the area of $D$ is the sum of the areas of $D_{1}, D_{2}$ and $D_{3}$, plus $2 \pi$. Hence, the area of $D$ is at least $2 \pi$. The case of equality is straightforward and is omitted.

\section{Levelling the interior edges}

In this section, we will prove the following result.

Theorem 6. Let $M$ be a once-punctured torus bundle with an angled polyhedral decomposition that is invariant under the fibre-preserving involution. Then there is an equivariant ambient isotopy taking each interior edge into a fibre.

Proof. We place a transverse orientation on the fibres, so that, locally, we may speak of one fibre being 'above' or 'below' another.

We may equivariantly ambient isotope the interior edges of $M$ so that the following conditions hold. Each interior edge either lies entirely in a fibre, or is transverse to the fibration at all but finitely many critical points, which are local maxima or minima. We may assume that the endpoints of each non-level interior edge are critical; in other words, their end tangents are horizontal. We may also assume that a fibre cannot contain both level edges and critical points, and that if a fibre contains more than one critical point or more than one level edge, then it contains precisely two and these are swapped by the involution. The fibres containing critical points or level edges we term critical. Critical fibres are divided into three types: 
- interior-critical, which contain an isolated critical point in the interior of $M$;

- boundary-critical, which contain an endpoint of a non-level edge;

- level-critical, which contain a level edge.

Define the weight of a critical fibre to be

- two, if the fibre is interior-critical and it contains two critical points (that must be swapped by the involution);

- the number of level edges it contains, if it level-critical;

- one, otherwise.

Let the width of $\Delta^{1}$ be the sum, over all critical fibres, of the product of the weight of the fibre and its number of transverse points of intersection with $\Delta^{1}$. Perform an equivariant ambient isotopy which minimises width. The 1-skeleton is then in thin position.

The reason for the weighting in the definition is as follows. Suppose that local maxima of $\Delta^{1}$, in distinct adjacent critical fibres, can be equivariantly isotoped past each other. Then we do not want this to alter the width of $\Delta^{1}$. This is the case with this choice of weighting. See Figure 6, for example.

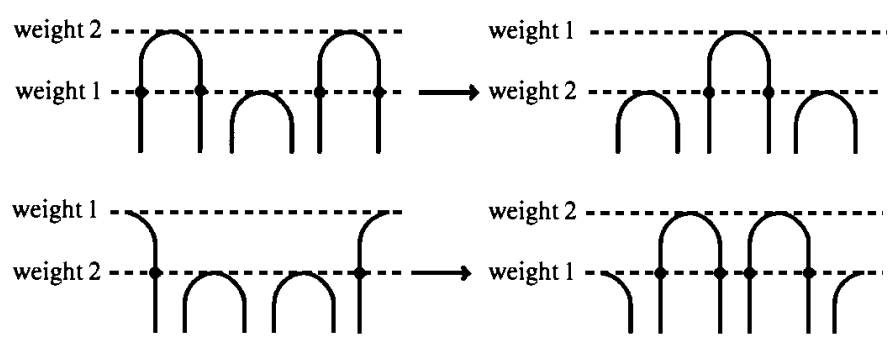

Figure 6 .

Suppose that not all the edges of $\Delta^{1}$ are level. Consider an interval $I$ of fibres, disjoint from the interior-critical and boundary-critical fibres, starting just below a local maximum for $\Delta^{1}$, and descending to just above a local minimum for $\Delta^{1}$. We may assume that each fibre in $I$, other than the level-critical fibres, has maximal intersection with $\Delta^{1}$ among all fibres not containing level edges. The interval $I$ is possibly divided up into sub-intervals by level-critical fibres. Consider one such sub-interval $I^{\prime}$, which we initially take to be the highest sub-interval. All fibres in this sub-interval are equivariantly ambient isotopic, leaving $\Delta^{1}$ invariant. Hence, by examining the supremal fibre, we see that each fibre in $I^{\prime}$ has a strict upper disc, which is defined as follows. An upper disc (respectively, lower disc) for a fibre $F$ is an embedded disc $D$ in $M$ such that

- its boundary is the union of an arc in $F$, an arc in $\Delta^{1}$ and possibly an arc in $\partial M$

- the above arcs of $D \cap \Delta^{1}$ and $D \cap \partial M$ are the only points of intersection between $D$ and $\partial M \cup \Delta^{1}$; 

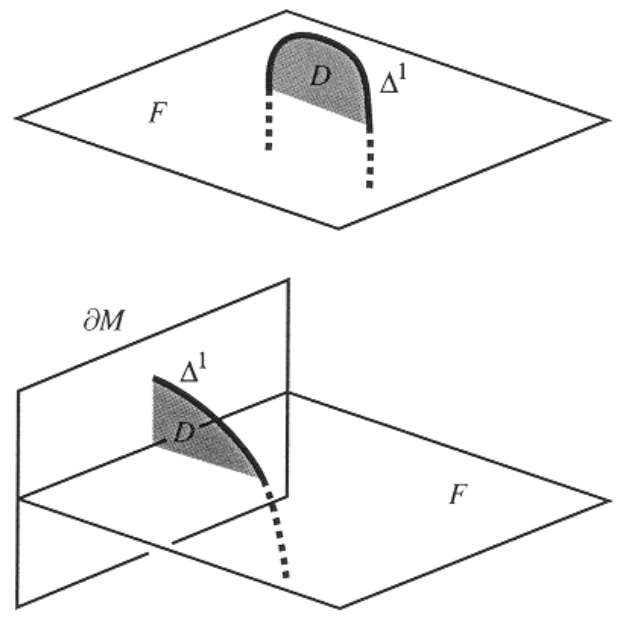

Figure 7 .

- near $\partial D \cap F$, its interior is disjoint from $F$, and it emanates from the upper (respectively, lower) side of $F$;

- $D$ and its image under the fibre-preserving involution are either equal or disjoint.

An upper or lower disc is strict if its interior is disjoint from $F$. Strict upper discs are shown in Figure 7.

If, directly below $I^{\prime}$, there is a level edge, then each fibre $F$ in $I^{\prime}$ has a lowerparallelity disc, which is an embedded disc in $M$ such that

- its boundary is an arc in $F$, an edge of $\Delta^{1}$ and two arcs in $\partial M$;

- its interior is disjoint from $\Delta^{1} \cup \partial M \cup F$;

- it is attached to the lower side of $F$;

- it and its image under the fibre-preserving involution are either equal or disjoint.

Upper-parallelity discs are defined similarly.

We define an upper disc and a lower disc for a fibre $F$ to be a thinning pair if they are disjoint away from $F \cap \Delta^{1}$ and the same is true of the upper disc and the image of the lower disc under the involution. The following results are easy generalisations of standard facts about thin position:

- No fibre can have a thinning pair of upper and lower discs.

- No fibre can have an upper disc contained in a lower disc or vice versa.

- If a fibre contains a level edge of $\Delta^{1}$, then it has no upper disc and no lower disc.

We will prove only the first of the above statements, as the remainder have similar proofs. Suppose that $U$ and $L$ are upper and lower discs that form a thinning pair. Consider the intersection between $U$ and the critical fibres, which we may 
assume is a sub-arc of $\partial U$, a finite collection of points on $\partial U$, properly embedded simple closed curves and properly embedded arcs that are disjoint except possibly at their endpoints. Let $D$ be a subdisc of $U$ separated off by an extrememost arc $\alpha$. If the endpoints of $\alpha$ are equal, there is an equivariant isotopy of $U$, keeping $\partial U$ fixed, that removes $\alpha$. Otherwise, we equivariantly isotope $D \cap \Delta^{1}$ across $D$ to remove $\alpha$, which does not increase the width of $\Delta^{1}$. We can isotope $L$ similarly. Thus, we may assume that $U$ and $L$ lie just above and just below a fibre. There is then an equivariant ambient isotopy that decreases the width of $\Delta^{1}$, either by moving the maximum of $U$ past the minimum of $L$ or by cancelling these critical points. This is impossible, since the 1-skeleton is in thin position.

Claim 1. In the interval $I^{\prime}$, some fibre $F$ intersects each interior face of $M$ transversely in the following components: simple closed curves, arcs with endpoints in $\partial M$, arcs with endpoints in distinct interior edges, and arcs with an endpoint in an interior edge and a non-adjacent boundary edge.

We must show that some fibre $F$ in $I^{\prime}$ intersects each face transversely and has neither of the following components of intersection with an interior face: an arc with endpoints in the same interior edge, or an arc running from an interior edge to an adjacent boundary edge. Note that, in each case, this arc separates off an upper or lower disc. We term these local upper and lower discs. It is impossible for any fibre to have both local upper and local lower discs, by thin position.

During the isotopy specified by the interval $I^{\prime}$, we may assume that $F$ intersects each interior face transversely, except at finitely points in $I^{\prime}$, where the intersection with any interior face performs the following moves:

- add or remove an innermost simple closed curve;

- add or remove an extrememost arc with both its endpoints in the same boundary edge;

- move two curves together (possibly the same curve) until they meet at a point, then resolve this singularity;

- move a curve towards a boundary edge, until it meets the edge at a point, then resolve this singularity;

- move the endpoints of two arcs in the same boundary edge towards each other until they meet, and then resolve this singularity by pulling them away from the boundary edge.

If the face is invariant under the involution, then two copies of a move may occur simultaneously in the face.

The first two moves do not affect the existence of local upper or lower discs. If two fibres differ by the third, fourth or fifth move, it is impossible for one to have a local lower disc and the other to have a local upper disc. For the non-transverse fibre between them would have both such discs, and a small isotopy would make these a thinning pair.

Now, the highest fibre of $I^{\prime}$ has an upper disc with interior disjoint from the interior faces. Hence, it cannot have a local lower disc. Similarly, the lowest fibre 
of $I^{\prime}$ cannot have a local upper disc. Hence, we deduce that some fibre $F$ in $I^{\prime}$ has no local lower and no local upper disc, which proves the claim.

Claim 2. There is an equivariant ambient isotopy, that is fixed on the interior edges, taking $F$ into fairly normal form.

We will perform a series of moves that will each reduce the number of components of intersection with the interior faces. Hence, they will eventually terminate.

Suppose that we were to perform equivariant compressions and boundarycompressions to the components of intersection between $F$ and the polyhedra, so that afterwards each such component is a disc that intersects each boundary face in at most one arc. The resulting surface we call $\bar{F}$. It is a collection of spheres and discs, together with a single once-punctured torus. The number of compressions and boundary-compressions used ( $n$, say) is equal to the number of spheres and discs. Each component of intersection between $\bar{F}$ and each polyhedron is either weakly normal, boundary-trivial or face-trivial. The area of each sphere and disc is negative, and so, by Lemma 4, it must contain a boundary-trivial or face-trivial disc. But a face-trivial or boundary-trivial disc can only be attached to another such disc. So, there are in total $2 n$ trivial discs in $\bar{F}$. The original surface $F$ is obtained from $\bar{F}$ by attaching $n$ tubes, at least one of which must be attached to the once-punctured torus component. Hence, if $n>0$, some trivial disc lies in $F$. We can perform an equivariant isotopy of $F$ to remove this disc. The resulting surface still satisfies Claim 1. Thus, we can repeat until $F$ is composed of weakly normal discs. If one of these discs fails to satisfy the last condition in the definition of being fairly normal, there is an obvious equivariant isotopy in the complement of the interior edges that reduces the number of intersections with the boundary faces. Finally, if a disc of $F$ intersects an interior edge more than twice, then a thinning pair of upper and lower discs can be found, contradicting thin position. This proves the claim.

Claim 3. If a fairly normal fibre $F$ has a strict upper disc, then it has a face compression disc on the upper side of $F$. If $F$ has an upper-parallelity disc, then either there is a face compression disc on the upper side of $F$, or this upperparallelity disc can be ambient isotoped into a polyhedron. Similar statements are true for discs below $F$.

Consider the intersection of the strict upper disc $U$ with the interior faces. We may assume that, near $\Delta^{1}$, the only intersection between $U$ and the interior faces is $\Delta^{1} \cap \partial U$. Thus, the intersection is $\Delta^{1} \cap \partial U$ and a collection of arcs and simple closed curves properly embedded in $U$, disjoint from $\Delta^{1}$. The closed curves may be removed. Consider an extrememost arc in $U$ away from $\Delta^{1} \cap \partial U$. If it has both endpoints in $\partial M$, it may be removed. The arc separates off a disc that lies in a polyhedron $P$. If this is not a face compression disc, then its intersection with $\partial P$ 
is parallel in the complement of $\Delta^{1}$ to an arc in $F \cap \partial P$. There is then an ambient isotopy of $U$, removing this arc of intersection. Repeating this process, we isotope $U$ into a polyhedron $P$. A further small isotopy in $P$ makes it a face compression disc.

The situation with an upper-parallelity disc $U$ is similar. The intersection between $U$ and the interior faces is again a collection of simple closed curves and arcs, and by the above argument, we may assume that it consists only of arcs running between distinct components of $U \cap \partial M$. Consider such an arc $\alpha$ in the disc that is closest (in $U$ ) to the edge of $\Delta^{1}$, separating off a sub-disc of $U$. This sub-disc forces $\alpha$ and the edge of $\Delta^{1}$ to be parallel in a polyhedron. We may therefore isotope $U$ to remove $\alpha$, and repeat until the parallelity disc lies entirely in a single polyhedron. This proves the claim.

So far we have not used the fact that $F$ is a once-punctured torus. We will do so now, by means of an area argument.

Claim 4. If a fairly normal fibre $F$ has a face compression disc on its upper side, then this is attached to an arclike disc with the arc on its upper side. A similar statement is true for a face compression disc below $F$.

Suppose that the face compression disc does not emanate from the arc side of an arclike disc. Then, by Lemma 5, the fairly normal disc to which it is attached has area at least $\pi$. Moreover, if it invariant under the involution, then it has area at least $2 \pi$. This disc and its image under the involution therefore account for all the area of $F$. By Lemma 5 , each is disjoint from $\Delta^{1}$, or is a modified or fused vertex link. The remaining discs have zero area, and hence by Lemma 4 , each is a bigon or vertex link.

Case 1. The discs with positive area are disjoint from $\Delta^{1}$.

A vertex link disc cannot be attached to a disc disjoint from $\Delta^{1}$. Therefore, there are no vertex link discs. So, $F$ is entirely disjoint from $\Delta^{1}$. But $F$ was, by construction, a fibre with maximal intersection with $\Delta^{1}$. This is a contradiction.

Case 2. The discs with positive area are modified vertex links.

A modified vertex link has arcs of intersection with interior faces that run from an interior edge to a boundary face. These arcs cannot be attached to a vertex link or to a bigon. So, this arc must be attached to a modified vertex link on the other side of the face. But this creates a component of $\partial F$ which bounds a disc in $\partial M$, which does not occur. Thus, this case does not arise.

Case 3. The only disc with positive area is a fused vertex link. 
A bigon cannot be attached to a fused vertex link or to a vertex link. So, $F$ consists only of a fused vertex link and vertex links. This implies that $F$ is closed, which is a contradiction, proving the claim.

We know that the fairly normal fibre $F$ in $I^{\prime}$ has a strict upper disc. Hence, by Claim 3, it has a face compression disc on its upper side. By Claim 4, it therefore has an arclike disc with the arc on the upper side. It cannot therefore have an arclike disc with the arc on the lower side. For this would imply that it has a thinning pair of lower and upper discs, contradicting thin position. So, $F$ has no face compression disc on its lower side. Now, either $F$ has a strict lower disc or there are level edges directly below $F$ in the fibration. In the former case, Claim 3 implies it has a face compression disc on its lower side, which we have already ruled out. So directly below $F$ there are level edges. These give rise to lower-parallelity discs $P$. Since $F$ has no face compression discs on its lower side, Claim 3 gives that the lower-parallelity discs can each be isotoped into a polyhedron.

Claim 5. $F$ has at most two arclike discs, and if it has exactly two, then these are swapped by the involution. Also, the lower-parallelity discs $P$ are attached to these arclike discs.

If not, then we can find an arclike disc $D$ disjoint from $P$. This arclike disc gives rise to an upper disc for the fibre containing the level edges in $P$. This contradicts thin position, and so establishes the claim.

The fibre $F^{\prime}$ directly below the level edges is obtained by isotoping $F$ across $P$. By Claim $5, F^{\prime}$ is fairly normal and has no arclike discs. (See Figure 8 .) So, directly below $F^{\prime}$, there cannot be a local minimum for $\Delta^{1}$, by Claims 3 and 4 . So, below $F^{\prime}$ there are more level edges, which give rise to further lower-parallelity discs. These can be each isotoped into a polyhedron. But this implies that the parallelity discs above $F^{\prime}$ are disjoint from those below $F^{\prime}$. They therefore extend to parallelity discs below $F$ that are disjoint from its arclike discs. Without changing the width, we may equivariantly isotope the level edges below $F^{\prime}$ and the level edges above $F^{\prime}$ past each other. This creates a fibre containing a level edge and having an upper disc, which contradicts thin position.

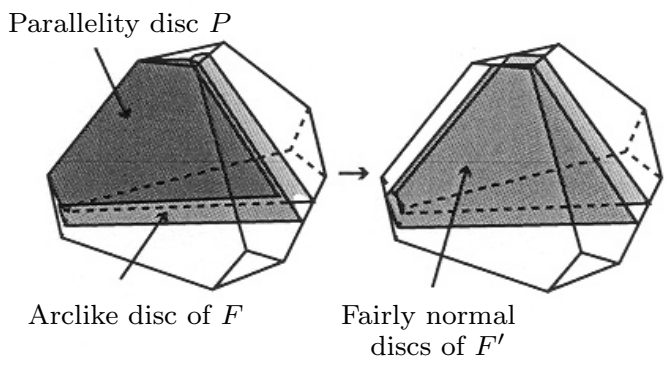

Figure 8 . 


\section{Conclusion of the proof}

Lemma 7. Every interior edge is invariant under the involution, and its orientation is reversed.

Proof. Each interior edge $e$ lies in a fibre which is preserved under the involution. This involution leaves invariant every properly embedded essential arc in the fibre, up to isotopy, and reverses its orientation. Thus, $e$ and its image either coincide or are parallel in the surface. However, it is impossible for distinct edges in a fibre to be parallel. For, the parallelity disc between two adjacent edges lies in the complement of $\Delta^{1}$. The intersection between this disc and the interior faces is a collection of properly embedded arcs and simple closed curves. By removing innermost curves and extrememost arcs, the disc may be isotoped into a polyhedron. We then see that $e$ must coincide with its image.

Proposition 8. The fixed point set of the involution cannot have an arc of intersection with any face.

Proof. Via standard Morse theory, we may apply an equivariant isotopy of the interior faces, keeping the interior edges fixed, so that the interior of each face has only finitely many critical points in the fibration. This gives a singular foliation on each face. We define the critical leaves to be those that contain critical points. So, the complement of the critical leaves has a product foliation. In the interior of the faces, we may take the critical points to have the following standard forms:

- saddles, which form a 4-valent vertex of a critical leaf;

- maxima and minima, whose corresponding critical leaf is a point.

We insist that each boundary edge is either level or has finitely many critical points of the following forms:

- maxima and minima, which create a critical leaf that is a point;

- half-saddles, which contribute 2-valent vertices to a critical leaf.

Near each level edge, we may arrange that each interior face has a finite number of switches, shown in Figure 11. We may assume that each fibre not containing level edges contains at most two critical points, and that if it does contain two, then these are swapped by the involution. We may also arrange that fibres containing level edges are disjoint from the saddle, half-saddle, maximum and minimum critical points.

We may remove all maxima and minima as follows, at the cost of introducing ridges (see Figure 12). These are properly embedded arcs in an interior face, with endpoints in $\partial M$, which are level in the fibration and form either the highest or lowest points in a neighbourhood of the arc. 

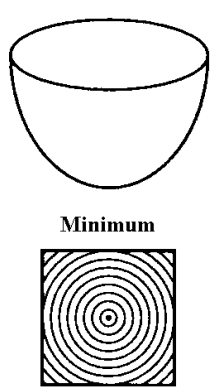

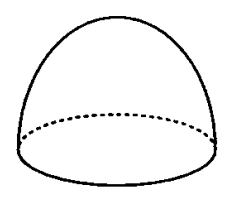

Maximum

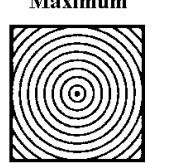

Figure 9.
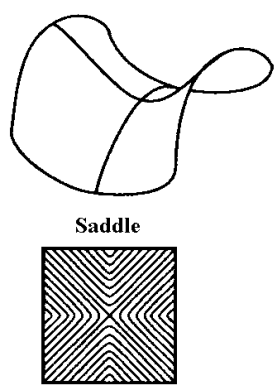
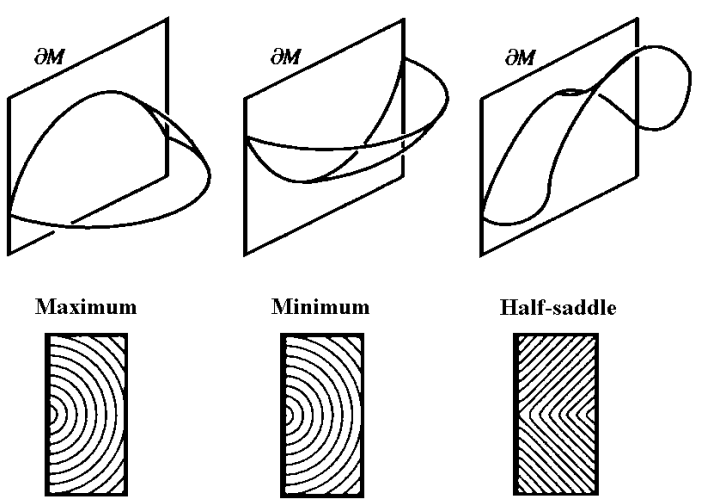

Figure 10.

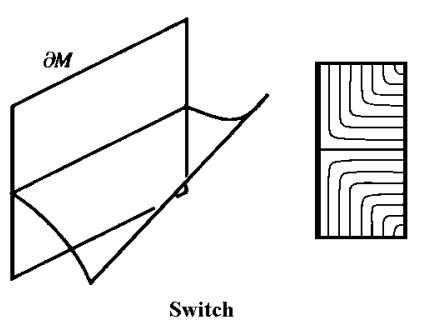

Figure 11.

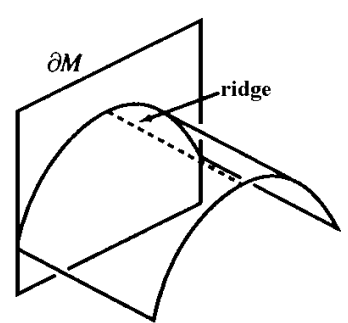

Figure 12. 


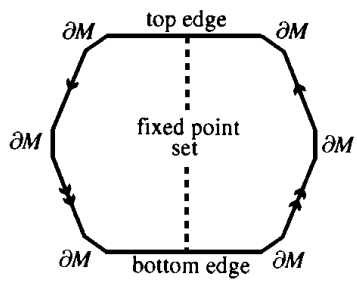

Figure 13.

For suppose that there is a local maximum, say. The adjacent complementary region $R$ of the critical leaves has a product foliation consisting of properly embedded arcs or circles, depending on whether the maximum lies in the boundary or the interior of $M$. Hence, $\partial R$ contains no other maxima or minima. So, it contains another type of critical point. There are a number of possibilities for the critical point (or points) in $\partial R$ and for the maximum. In each case, we can cancel the maximum with a critical point (or points). For example, if the maximum lies on the boundary of $M$, and the other critical point is a half-saddle, then these may be cancelled to form a ridge. The only small complication is when the maximum is in the interior of the face and there is a single critical point in $\partial R$ that is a saddle. It may be the case that $\partial R$ runs over this saddle twice. But then $R$ separates off a subdisc of the face, which contains at least one maximum or minimum. So, by passing to an innermost region, we can assume this case does not arise. Thus, we may ensure that each face now has no maxima or minima. This implies that the complement of the critical leaves is foliated by arcs, and that each complementary region has two critical leaves in its boundary. The region can be viewed as a square in which the height monotonically descends from one critical leaf to the other.

Suppose now that a face contains an arc of intersection with the fixed point set of the involution. Then, the face is subjected to a rotation about this arc. Since each interior edge is reversed by the involution, the gluing pattern of the edges of the face must be as shown in Figure 13, possibly with further identifications. The arc of the fixed point set has endpoints in two interior edges of the face, the 'top' and 'bottom' edges, $t$ and $b$. Consider the complementary region $R$ of the critical leaves attached to the part of $t$ that intersects the fixed point set, and consider the critical leaf in $\partial R$ not intersecting $t$. Its intersection with the fixed point set is not a critical point, since the fixed point set is transverse to the fibration. Hence, emanating from this point is a sub-arc $\alpha$ of the critical leaf. There are the following possibilities for the next critical points along this arc:

1. $\alpha$ is properly embedded in the face and ends in switches.

2. $\alpha$ ends in saddles.

3. $\alpha$ ends in half-saddles.

4. $\alpha$ contains a subset of the bottom edge $b$.

Let us consider Case 1. The switches lie in interior edges $e$ and $e^{\prime}$, neither of 

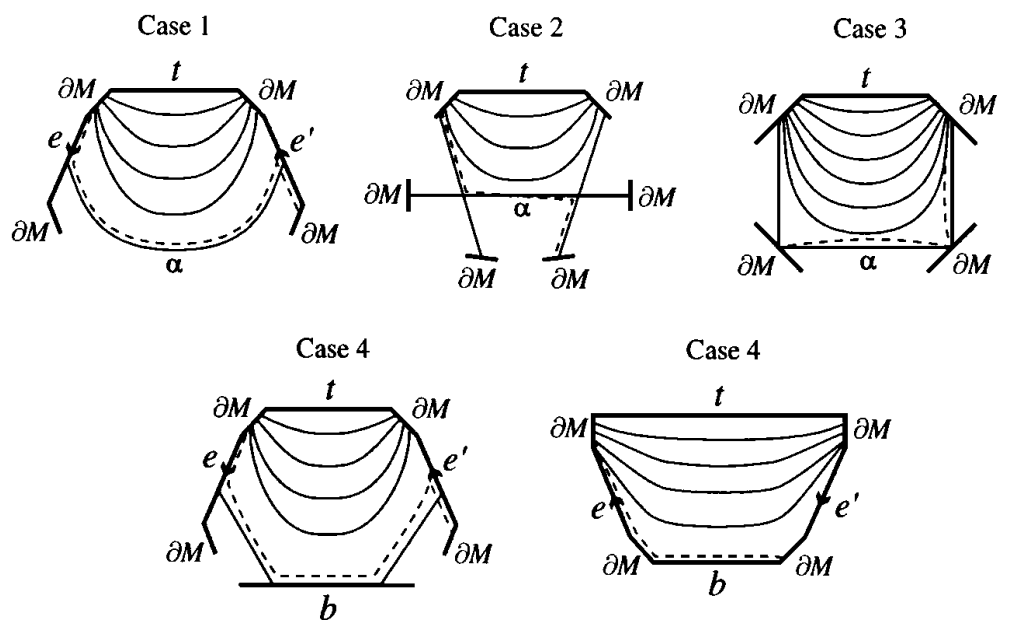

Figure 14.

which can be $t$ or $b$. These must be the same edge in $M$, since each interior edge is preserved under the involution. The arc $\alpha$ and the edge $e=e^{\prime}$ both lie in the same fibre $F$. Consider the arc $\beta$ in the face that starts in $\partial M$, runs forwards along $e$ as far as an endpoint of $\alpha$, then runs along $\alpha$, then runs backwards along $e^{\prime}$ back to $\partial M$. This is shown in Case 1 of Figure 14 as a dotted arc. Since $e$ is an essential embedded arc in $F$ and the interior of $\alpha$ is embedded, $\beta$ is homotopic in $F$ to a properly embedded arc. Hence, it and its image under the involution are homotopic in $F$ as unoriented arcs. Thus, we deduce that $\beta$ and its image are homotopic in $M$ via a homotopy whose interior misses $\Delta^{1}$. However, this is impossible, by the following claim, since $\beta$ and its image are distinct essential arcs in the face, not parallel to an interior edge. The proof of this claim is simple and omitted.

Claim. Suppose that two properly embedded arcs in interior faces are homotopic, via a homotopy that misses $\Delta^{1}$. Then either these are parallel arcs in the same face, or they are both parallel in the faces to interior edges, or they are both inessential in their faces.

Cases 2 and 3 are dealt with similarly. In each case, we apply the argument of Case 1 to the dotted arcs in Figure 14. Consider now Case 4. The non-singular leaves in $R$ end in boundary edges. These cannot be adjacent to both $t$ and $b$. Suppose that they are not adjacent to $b$. Then, emanating from $b$ in the boundary of $R$, there are either switches or level boundary edges. In the former case, the critical leaf continues from the switches to other interior edges $e$ and $e^{\prime}$, which are swapped by the involution. We can apply the argument of Case 1 to the dotted 
arc shown in Figure 14. In the latter case, the level boundary edges are attached to other interior edges $e$ and $e^{\prime}$. To apply the argument of Case 1 to the dotted arc in Figure 14, we need to know that it is homotopic in its fibre $F$ to a properly embedded arc. This is clear if $e$ and $b$ are distinct interior edges. We claim that they cannot be the same edge. Suppose that they were. If the orientations of $e^{\prime}$, $b$ and $e$ were all consistent around $\partial R$, then their concatenation would not be a primitive element of $H_{1}(F, \partial F)$, and hence would not be homotopic to a properly embedded arc. However, their concatenation is homotopic to such an arc, as can be seen by considering a nearby non-singular leaf. If the orientations of $e$ and $b$ are inconsistent around $\partial R$, then the level edge between them would have to start and end at the same point, and hence would close up to form a level closed curve in $\partial M$. But, it and its image under the involution have disjoint interiors, which would then not be possible. So, we can again apply the argument of Case 1.

Denote the number of polyhedra by $p$, the number of interior faces by $f$ and the number of interior edges by $e$. When the fixed point set is cut along the points of intersection with the 2-skeleton, the result is $s$ arcs, say. We have the following inequalities:

- $p \geq s$, since each polyhedron can contain at most one arc of the fixed point set in its interior. This is an equality if and only if each polyhedron is invariant under the involution.

- $s \geq e$, as Lemma 7 implies that each interior edge intersects the fixed point set, and Proposition 8 gives that no face contains an arc of intersection with the fixed point set. This is an equality if and only if the fixed point set is disjoint from the interior of the faces.

- $e \geq p$, as each polyhedron has at least four faces, and each interior face lies on the boundary of two polyhedra (or forms two faces of a single polyhedron). Hence, $2 f \geq 4 p$. But by Euler characteristic, $e-f+p=0$ and so this gives the required inequality. This is an equality if and only if each ideal polyhedron is an ideal tetrahedron.

We then deduce that the above inequalities must be equalities. Therefore, each polyhedron is a tetrahedron that is preserved by the involution. The fixed point set must run through every tetrahedron exactly once between the midpoints of opposite edges. The interior faces of each tetrahedron are partitioned into two orbits, and the two faces in an orbit form a square with side identifications. The involution applies an order two rotation to this square, and reverses the orientation of each of the interior edges. Hence, the side identifications of the square are those of a once-punctured torus. We therefore see that the ideal triangulation is obtained from a copy of the once-punctured torus by successively attaching ideal tetrahedra, realizing elementary moves, and then gluing top to bottom. In this sequence of moves, no move can be immediately followed by its inverse, as this would create an edge with valence two, which is impossible in angled polyhedral decomposition. Hence, this is the monodromy ideal triangulation of a representation of $M$ as a once-punctured torus bundle. However, $M$ fibres over the circle in only one way, 
since it easy to check that $H_{2}(M, \partial M) \cong \mathbb{Z}$. As the space of ideal triangulations of the punctured torus is a tree, there is only one possible such sequence of moves that realizes the monodromy. Thus, this is the monodromy ideal triangulation of $M$ for the given bundle structure. This proves Theorems 1, 2 and 3.

\section{References}

[1] H. Akiyoshi, M. Sakuma, M. Wada and Y. Yamashita, Ford domains of punctured torus groups and two-bridge knot groups, in: Knot Theory, Conference Proceedings, Toronto, 1999.

[2] D. B. A. Epstein and R. C. Penner, Euclidean decomposition of non compact hyperbolic manifolds, J. Differential Geom. 27 (1988), 67-80.

[3] W. Floyd and A. Hatcher, Incompressible surfaces in punctured torus bundles, Topology Appl. 13 (1982), 263-282.

[4] D. Gabai, Foliations and the topology of 3-manifolds III, J. Differential Geom. 26 (1987), $479-536$.

[5] T. Jørgensen, On pairs of once-punctured tori, unfinished manuscript.

[6] M. Lackenby, Word hyperbolic Dehn surgery, Invent. Math. 140 (2000), 243-282.

[7] I. Rivin, On geometry of convex ideal polyhedra in hyperbolic 3-space, Topology 32 (1993), $87-92$.

[8] J. H. Rubinstein, Polyhedral minimal surfaces, Heegaard splittings and decision problems for 3-dimensional manifolds, Proceedings of the Georgia Topology Conference, AMS/IP Stud. Adv. Math, vol. 21, Amer. Math Soc. (1997) 1-20.

[9] M. Stocking, Almost normal surfaces in 3-manifolds, Trans. Amer. Math. Soc. 352 (2000), $171-207$.

[10] A. Thompson, Thin position and the recognition problem for the 3-sphere, Math. Res. Lett. 1 (1994), 613-630.

[11] J. Weeks, SnapPea, available at http://thames.northnet.org/weeks/index/

Marc Lackenby

Mathematical Institute

Oxford University

24-29 St Giles'

Oxford OX1 3LB

England

(Received: January 4, 2002) 